\title{
LES STRUCTURES ARGYROPHILES SUPERFICIELLES DES FORMES LARVAIRES INFESTANTES DE SCHISTOSOMA JAPONICUM
}

\author{
J.-L. ALBARET*, Ch. BAYSSADE-DUFOUR* et M. TSUJI**
}

\begin{abstract}
RÉSUMÉ. Les structures argyrophiles tégumentaires du miracidium et de la cercaire d'une souche chinoise de Schistosoma japonicum sont décrites en détail.

- Miracidium. La disposition des organites au niveau des ceintures antérieure et moyenne est identique à celle du miracidium de Schistosoma incognitum mais distingue très nettement ces deux miracidiums de ceux des autres espèces de Schistosoma.

- Cercaire. L'imprégnation argentique montre des différences dans la chétotaxie au niveau du corps cercarien par rapport à la seule description faite antérieurement mais il s'agit, à notre avis, de différences d'interprétation et non de différences de structures. Le nombre réduit des récepteurs sensoriels caudaux permet une distinction aisée entre la cercaire de $S$. japonicum et celles des autres espèces du genre.
\end{abstract}

\section{Superficial argentophilic structures of miracidium and cercaria of Schistosoma japonicum}

SUMMARY. Integumentary argentophilic structures in miracidium and cercaria of Schistosoma japonicum, chinese strain, are described in detail.

- Miracidium. The pattern of organites between the first and second tier and between the second and third tier in miracidia of $S$. japonicum is identical to that in miracidia of $S$. incognitum, but differs from that seen in miradicia of other species of Schistosoma.

- Cercaria. Chaetotaxy differs from that reported in a previous study but, in our opinion, these differences concern the interpretation of the authors and not the structure of the cercaria.

$S$. japonicum is clearly distinguished from other species in the genus by the reduced number of sensory receptors on the tail.

Schistosoma japonicum est un parasite anthropo-zoophile largement répandu en Extrême-Orient et particulièrement pathogène pour l'Homme.

Cette espèce est représentée par au moins 4 races géographiques susceptibles d'évoluer chez 5 sous-espèces du Mollusque Prosobranche Oncomelania hupensis (Hunter et coll., 1952 ; De Witt, 1954; Hsü et Hsü, 1960 ; Davis 1969; Davis et Carney, 1973).

* Muséum National d'Histoire Naturelle, Laboratoire de Zoologie-Vers associé au C.N.R.S., 61 rue de Buffon, F 75231 Paris Cedex 05.

** Chaire de Parasitologie, Faculté de Médecine. Université de Hiroshima Kasumi 1-2-3, Hiroshima 734 Japon.

Accepté le 8 janvier 1982 
L'étude des structures argyrophiles superficielles des formes larvaires infestantes a fait l'objet des travaux d'Ozaki (1952) et Sakamoto et Ishii (1978). Le premier auteur s'est adressé au miracidium d'une souche dont l'origine géographique et le Mollusque vecteur ne sont pas précisés. Il donne une description des organites argyrophiles de la seule ceinture antérieure. Les seconds fournissent une description détaillée des récepteurs sensoriels de la cercaire d'une souche vraisemblablement japonaise émise par Oncomelania hupensis nosophora.

Disposant d'une souche passant par Oncomelania hupensis hupensis, il nous a été possible de compléter les résultats d'Ozaki et de comparer les miracidiums à ceux d'autres espèces du genre. De plus, nous avons pu observer au niveau du corps cercarien des différences dans le nombre des papilles ventrales par rapport à la description de Sakamoto et Ishii. Enfin l'étude chétotaxique permet de distinguer aisément les cercaires de S. japonicum de celles des autres espèces du genre Schistosoma.

\section{Matériel et techniques}

La souche utilisée, isolée à partir d'un malade, dans la région de Shangaï (Chine) a fait l'objet de deux passages successifs sur Oncomelania hupensis hupensis - Souris blanche. Les cercaires obtenues après un troisième passage sur le même Mollusque ont servi en partie pour l'étude chétotaxique, en partie pour l'infestation d'un lot de Souris blanches. Après broyage du foie et de l'intestin de celles-ci, de nombreux miracidiums ont été obtenus.

Une émission notable de cercaires (environ 50 par Mollusque) n'a été possible qu'en alternant deux périodes d'éclairement (température $27^{\circ} \mathrm{C}$ ) avec deux périodes de mise à l'obscurité des Mollusques (température $20^{\circ} \mathrm{C}$ ) chaque période ayant une durée de 45 à 60 minutes.

Les miracidiums ont été imprégnés par la technique de Lynch (1933) et montés dans la gomme au chloral de Faure. L'imprégnation des cercaires a été effectuée avec la technique de Combes et coll. (1976).

\section{Le miracidium}

\section{DESCRIPTION}

- Cellules épidermiques ciliées (fig. 1, A)

Les cellules ciliées sont classiquement réparties sur quatre étages séparés par trois ceintures transversales : antérieure, moyenne et postérieure. Sur 92 miracidiums observés 79 portent 22 cellules ciliées avec, d'avant en arrière, la séquence : $6,9,4,3$. Les 13 autres miracidiums sont caractérisés par des variations du nombre des cellules ciliées à l'exception du premier étage. Le nombre de ces cellules peut être de 8 ou 10 pour le deuxième étage, de 3 ou 5 pour le troisième et de 2 ou 4 pour le quatrième. 

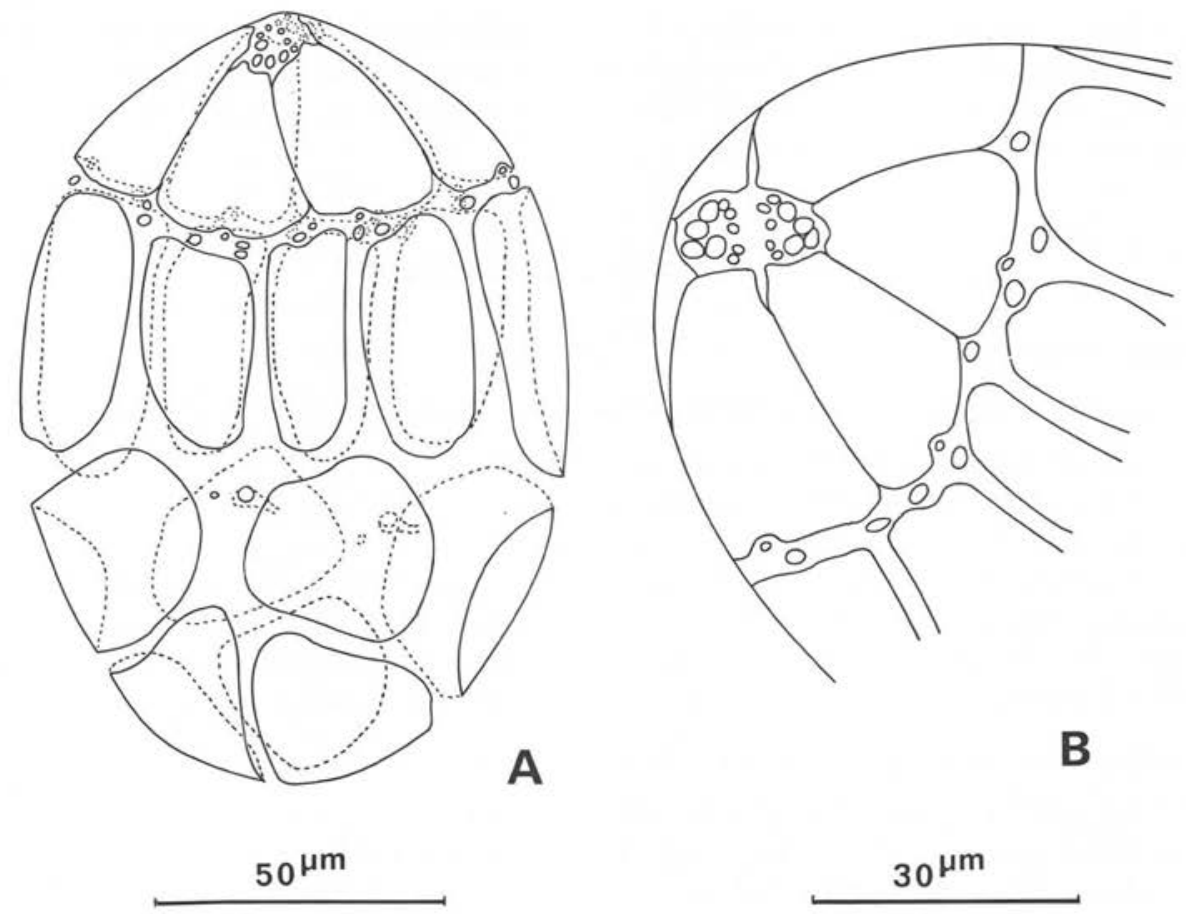

FIG. I. - Schistosoma japonicum. Miracidium : Structures argyrophiles superficielles.

A : vue latérale ; $B$ : vue apicale.

\section{- Organites argyrophiles superficiels (fig. 1, A et B)}

- Le térébratorium porte 9 formations argyrophiles par hémicorps, soit 4 formations d'un diamètre de $3 \mu \mathrm{m}$ et 5 formations plus petites disposées en 2 groupes de 2 contiguës et 1 isolée.

- La ceinture antérieure. Pour les 92 miracidiums observés le nombre total des organites varie de 13 à 28 (moyenne 25, 51) dont 6 papilles ciliées situées chacune dans une échancrure au milieu de la base de chaque cellule ciliée du premier étage.

- La ceinture moyenne ne porte que deux formations de très petit diamètre localisées chacune latéralement au voisinage des pores excréteurs.

Comparaison avec la description d'Ozaki

Le nombre et la disposition générale des cellules épidermiques ciliées sont conformes à la description d'Ozaki (1952). Toutefois le pourcentage de miracidiums présentant la séquence $6,9,4,3$ est un peu supérieur $(85 \%)$ à celui observé par cet auteur. En outre ce dernier signale des variations du nombre des cellules ciliées du premier étage alors que nous l'avons toujours trouvé constant. 
En ce qui concerne les organites argyrophiles nos observations apportent des précisions quant au nombre de ces éléments au niveau de la ceinture antérieure et elles fournissent des données nouvelles sur les organites argyrophiles du térébratorium et de la ceinture moyenne.

\section{La Cercaire : chétotaxie}

\section{OBSERVATIONS}

- Région céphalique par hémicorps (23 cercaires examinées) (fig. 2, $A, B$ et $C$ )

Le nombre des papilles est constant.

Le cycle $C_{I}$ comporte $1 C_{I} V, 8 C_{I} L$ et $1 C_{I} D$.

Le cycle $\mathrm{C}_{\mathrm{II}}$ se compose d'une $\mathrm{C}_{\mathrm{II}} \mathrm{V}$ et $1 \mathrm{C}_{\mathrm{II}} \mathrm{D}$.

Le cycle $\mathrm{C}_{\mathrm{III}}$ est constitué d'une $\mathrm{C}_{\mathrm{III}} \mathrm{V}, 1 \mathrm{C}_{\mathrm{II}} \mathrm{L}$ et $2 \mathrm{C}_{\mathrm{II}} \mathrm{D}$. Leur disposition présente quelques variations. Si dans 11 cas elles sont alignées transversalement ( $f$ ig. $2 \mathrm{~B}$ ) elles peuvent également être disposées obliquement ou en tandem ( $f i g .2 \mathrm{C}$ ). Ces variations peuvent affecter l'un ou l'autre hémicorps ou les deux.

- Région corporelle par hémicorps (28 cercaires examinées) (fig. $2 \mathrm{D}$ et E).

Le nombre des papilles est également constant.

Le cycle $A_{1}$ se compose d'une $A_{I} V$ de $3 A_{I} L$ et d'une $A_{I} D$.

Le cycle $\mathrm{A}_{\mathrm{II}}$ comporte $1 \mathrm{~A}_{\mathrm{II}} \mathrm{L}$ et $1 \mathrm{~A}_{\mathrm{II}} \mathrm{D}$.

Le cycle $A_{\text {II }}$ est formé d'une $A_{\text {II }} \mathrm{L}$ et $1 \mathrm{~A}_{\mathrm{II}} \mathrm{D}$.

Le cycle $\mathrm{A}_{\mathrm{IV}}$ comprend $1 \mathrm{~A}_{\mathrm{IV}} \mathrm{L}$ et $1 \mathrm{~A}_{\mathrm{IV}} \mathrm{D}$.

Le cycle $\mathrm{M}$ est constitué d'une ML.

Les cycles $P_{I}, P_{I I}$ et $P_{I I I}$ se composent respectivement d'une $P_{I} V, 1 \quad P_{I I} V$, $1 \mathrm{P}_{\mathrm{III}} \mathrm{V}$ et $1 \mathrm{P}_{\mathrm{I}} \mathrm{D}$.

- Acétabulum (28 cercaires examinées) (fig. $2 \mathrm{D}$ ).

On observe la disposition classique $\mathrm{S}=3 \mathrm{~S}_{\mathrm{I}}$ et $1 \mathrm{~S}_{\mathrm{II}}$.

- Quene (24 cercaires examinées) (fig. $2 \mathrm{D}$ et E).

Le tronc caudal porte au total 6 à 9 UV, 2 UL et 6 à 9 UD.

Chaque furca porte le plus souvent une papille. Celle-ci peut parfois faire défaut sur une des furcas ou plus rarement sur les deux.

Comparaison avec la description de Sakamoto et Ishii

La comparaison de la chétotaxie de la cercaire étudiée avec celle de la cercaire décrite par Sakamoto et Ishii (1978) (Cercaires émises par Oncomelania nosophora infestés par des miracidiums issus d'œufs provenant d'un Lapin), met en évidence une différence importante concernant les papilles ventrales avec la présence chez cette dernière cercaire de deux papilles supplémentaires par hémicorps ( 9 au lieu de 7) (fig. $2 \mathrm{~F}$ ). Or, nous pensons qu'une telle différence ne reflète pas la réalité et pourrait provenir du fait que les auteurs japonais ont pris pour les papilles $V_{5}$ et $V_{6}$ des 

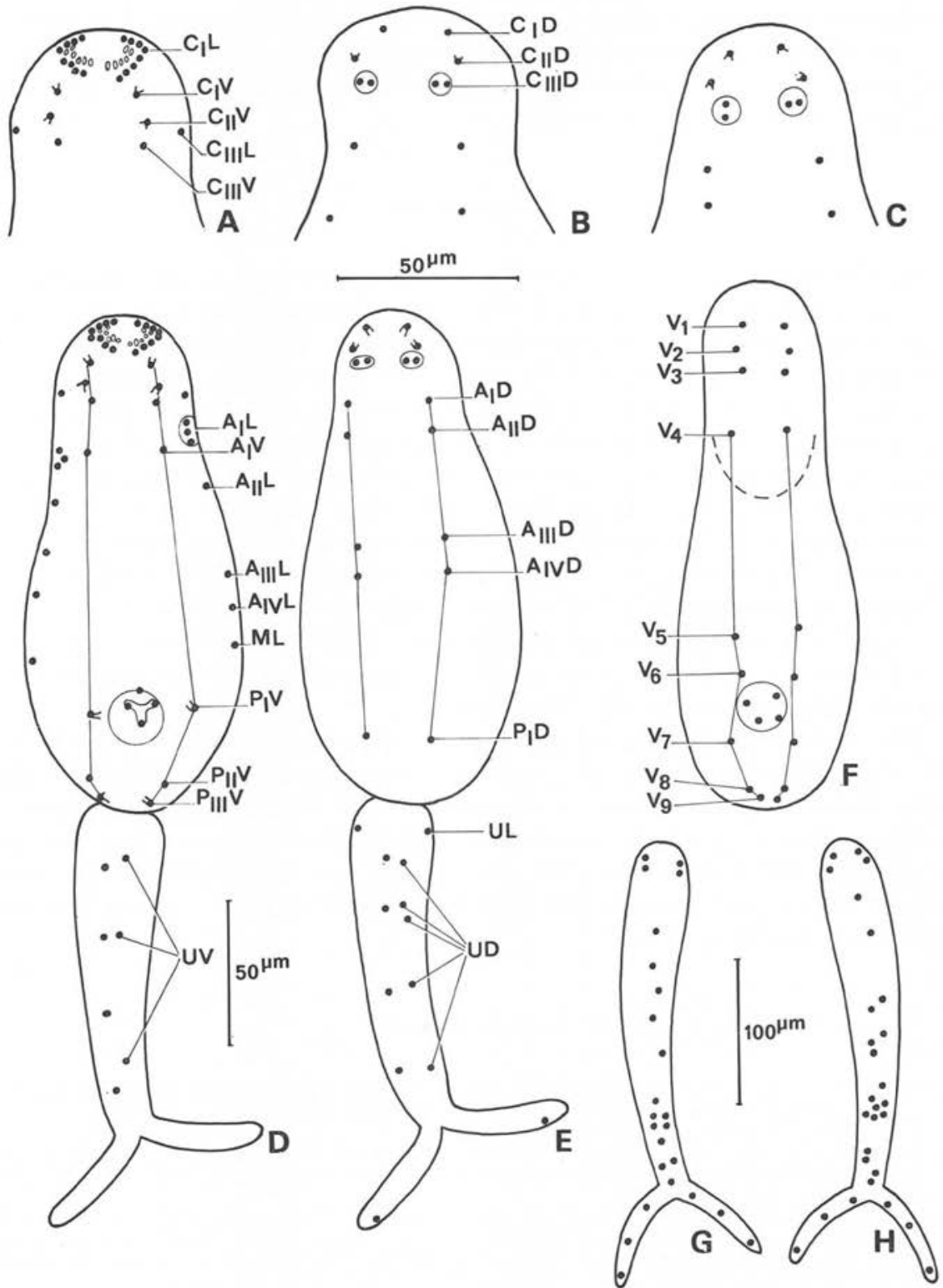

Fig. 2. - Schistosoma japonicum. Cercaires. Chétotaxie.

$\mathrm{A}$ : vue céphalique ventrale; $\mathrm{B}, \mathrm{C}$ : vues céphaliques dorsales; D : vue ventrale; $\mathrm{E}$ : vue dorsale ; $\mathrm{F}$ : corps cercarien, vue ventrale d'après Sakamoto et Ishii (1978); G et H : chétotaxie caudale des cercaires des autres espèces du genre Schistosoma; G : vue ventrale ; H : vue dorsale. 
granulations argyrophiles particulièrement abondantes à ce niveau. Un argument en faveur de cette hypothèse nous a été fourni par l'étude de la chétotaxie de cercaires émises par Oncomelania h. nosophora pour lesquelles le nombre des papilles est identique à celui de la cercaire étudiée.

\section{Conclusion}

Si l'on tient compte de l'ensemble des structures argyrophiles superficielles, nous constatons que le miracidium de Schistosoma japonicum est identique à celui de S. incognitum parasite de Mammifères (Chien, Porc, Muridae) décrit en Inde par Dutt et Srivastava (1961) mais diffère totalement au niveau des ceintures antérieure et moyenne des miracidiums des autres espèces du genre : $S$. mansoni décrit par Capron et coll. (1965) et Albaret (1978), S. haematobium décrit par Capron et coll. (1965), S. intercalatum décrit par Bayssade-Dufour et coll. (1980), S. spindale, $S$. nasale et $S$. indicum décrits par Dutt et Srivastava (1961).

D'autre part, la présence d'un nombre un peu inférieur de papilles sur le corps cercarien et l'existence d'un nombre réduit de papilles caudales distinguent très nettement les cercaires de S. japonicum de celles des Schistosoma de Planorbes : S. mansoni et S. rodhaini décrites par Richard (1971) et des Schistosoma de Bulins ; $S$. haematobium et $S$. bovis décrites par le même auteur et $S$. intercalatum décrite par Bayssade-Dufour et coll. (1980) (fig. $2 \mathrm{G}$ et $\mathrm{H}$ ).

Nos observations portent à quatre le nombre des espèces pour lesquelles les organites argyrophiles superficiels sont connus avec précision à la fois chez le miracidium et chez la cercaire : S. mansoni, S. haemotobium, S. intercalatum et S. japonicum. Or nous remarquons que la première espèce présente par rapport aux deux suivantes, des caractères différentiels nets mais discrets alors que le miracidium et la cercaire de Schistosoma japonicum sont d'un type franchement différent de ceux des autres Schistosomes humains.

\section{BIBLIOGRAPHIE}

Albaret J.-L. : Variations du nombre des papilles ciliées chez les miracidiums de différentes souches de Schistosoma mansoni agent de la bilharziose intestinale. C. R. Acad. Sci., I978, 286, sér. D, I359-I 362 .

Bayssade-Dufour Ch., Albaret J.-L., Picot H., Deniau M. : Les organites argyrophiles superficiels des formes larvaires infestantes de Schistosoma intercalatum. Ann. Parasitol. Hum. Comp., I980, 55, 249-252.

Capron A., Deblock S., Biguet J., Clay A., Adenis L., Vernes A. : Contribution à l'étude expérimentale de la bilharziose à Schistosoma haematobium. Bull. O.M.S., I965, 32, 755-778.

Combes C., Bayssade-Dufour Ch., CAssone J. : Sur l'imprégnation et le montage des cercaires pour l'étude chétotaxique. Ann. Parasitol., Hum. Comp. I976, sI, 399-400.

Davis G. M. : Oncomelania and the transmission of Schistosoma japonicum : a brief review. In : Harinasuta, C. (Ed.), Proceedings of the Fourth Southeast Asian Seminar on Parasitology and Tropical Medicine, Schistosomiasis and other Snail-transmitted Helminthiasis, Manila, $24-$ 27 February 1969. SEAMEC Central Coordinating board for Tropical Medicine and Public Health project, Bangkok, Thailand, 1969, 93-I03.

Davis G. M., CARNEy W. P.: Description of Oncomelania hupensis lindoensis, first intermediate host of Schistosoma japonicum in Sulawesi (Celebes). Proc. Acad. Nat. Sci. Philadelphia I973, I25, I-34. 
DE WrTt W. B. : Susceptibility of snail vectors to geographic strains of Schistosoma japonicum. J. Parasitol., 1954, 40, 453-456.

DutT S. C. SRIVASTAVA H. D. : On the epidermal structures of miracidia of six species of mammalian schistosomes and a new technique of specific diagnosis of animal schistosomiasis Indian J. Helminth, I96I, I3, roo-III.

Hsu S. Y. L., Hsu H. F. : Infectivity of the Philippine strains of Schistosoma japonicum in Oncomelania hupensis, O. formosana and O. nosophora. J. Parasitol., 1960, 46, 793-796.

Hunter G. W., Ritchie L. S., Otori Y. A. : Comparison of the infectivity of Schistosoma japonicum occuring in Japan for Oncomelania nosophora and Oncomelania formosana. J. Parasitol., 1952, 38, 492 .

LYNCH J. E. : The miracidium of Heronimus chelydrae, Mac Callum, Quart. J.Microsc. Sci., I933, $76, \mathrm{x} 3-33$.

OzAKI Y. : Epidermal structure of the miracidium of Schistosoma japonicum. (Katsurada). Annot. Zool. Jap., r952, 25, 343-35I.

Richard J. : La chétotaxie des cercaires. Valeur systématique et phylétique. Mém. Mus. nat. Hist. nat., nelle sér. sér. A. Zool., 1971, 67, 179 p.

SAKAмото K., IshiI Y. : Scanning electron microscope observations on miracidium, cercaria and cercarial papillar patterns of Schistosoma japonicum. J. Parasitol., 1978, 64, 59-68. 